\title{
RECHERCHE D'UNE TECHNIQUE DE TRANSPLANTATION DES EUFS CHEZ LA VACHE
}

\author{
J. TESTART et P.-C. LÉGLISE \\ avec la collaboration de F. Aymer de LA chevalerie \\ Institut national de la Recherche agronomique \\ Station centrale de Physiologie animale, 78-Jouy-en-Josas, France
}

De nombreux chercheurs ont tenté d'induire une gestation chez la Vache, après transplantation d'œufs fécondés, en empruntant deux voies d'accès à l'utérus :

La voie non chirurgicale qui consiste, comme pour l'insémination artificielle, à franchir le cervix, via le vagin, avec une pipette de transplantation, a donné des résultats médiocres.

DzIUK et al. (I958), AVERY et al. (I962) ne rapportent aucun succès sur un total de 28 essais. Les premiers, MUTTER, GRADEN et OLDS (I965) obtiennent une gestation à terme en perfusant le tractus de la vache receveuse avec une solution antibiotique depuis l'œestrus jusqu'au jour du transfert. Puis, Sugre (I965) obtient deux gestations, dont l'une à terme en perforant la paroi vaginale pour atteindre l'utérus en évitant le passage transcervical; de plus, il insuffle la corne utérine avec du gaz carbonique lors du transfert.

Rowson et MOOR (I966), puis VINCENT, MiLLS et RUNDELL (I969), reprenant la voie transcervicale mais en insufflant l'utérus avec du $\mathrm{CO}_{2}$, obtiennent chacun deux gestations sur 8 et I 5 vaches dont les cycles sont synchronisés à I jour près avec ceux des donneuses.

La voie chirurgicale a donné de meilleurs résultats. Après UmbaUGH (I949) qui provoqua 4 gestations mais qui n'allèrent pas jusqu'à leur terme, WILLE'T et al. (I95I) réussit à obtenir le premier veau après 3 essais, puis WILI,ET, BUCKNER et I,ARSON (I953) deux autres naissances pour deux tentatives. AVERY et al. (r962) rapportent la réussite d'une gestation à terme après quatre essais et récemment, Rowson, Moor et LAwson (I969), en récupérant les cufs des donneurs in vivo et en utilisant le "TC I99" comme milieu de perfusion et de conservation, indiquent un taux de réussite très élevé : $9 \mathrm{I}$ p. Ioo de receveuses gravides.

Ceci montre que le transfert des œufs par la voie abdominale, chez la Vache, est parfaitement possible puisque ce résultat est parmi les meilleurs obtenus chez l'ensemble des Mammifères. Mais l'utilisation d'une telle technique ne semble pas réalisable dans la pratique courante puisqu'elle exige, outre un matériel important et 
du personnel hautement spécialisé, des conditions opératoires (anesthésie, asepsie) et des durées d'intervention qui ne permettent pas son utilisation sur une grande échelle.

Aussi avons-nous répété les diverses tentatives de transfert non chirurgical pour en vérifier les résultats, tout en recherchant une technique qui utiliserait l'avantage de la voie transabdominale en évitant les servitudes d'une véritable intervention chirurgicale.

\section{I. - COMPARAISON DE TECHNIQUES DE TRANSPLANTATION DES GEFS CHEZ I,A VACHE}

Nous avons expérimenté diverses voies d'introduction des œufs dans la corne utérine, en transférant non des oufs de vache (pour limiter le nombre d'animaux utilisés), mais des œufs de Lapine, divisés ou non, ou des boules de résine de $200 \mu$ de diamètre environ ou des colorants.

\section{A. - Inovulation par le col de l'utérus}

\section{Méthode dérivée de l'insémination artificielle.}

Une sonde souple est guidée par palper transrectal, à travers le col et jusqu'au milieu de la corne. A travers la sonde un fin cathéter permet d'introduire les œufs in utero.

Le tableau I résume nos interventions.

TABLEAU I

Essais d'introduction d'cufs de lapine ou de billes de résine $(200 \mu)$ dans l'utérus de la Vache par le col de l'utérus

\begin{tabular}{|c|c|c|c|c|c|}
\hline \multirow{2}{*}{ Numéro de la vache } & \multicolumn{2}{|c|}{$\begin{array}{l}\text { Nombre de corps } \\
\text { transplantés }\end{array}$} & \multirow{2}{*}{$\begin{array}{c}\text { Délai } \\
\text { transplantation- } \\
\text { abattage }\end{array}$} & \multicolumn{2}{|c|}{$\begin{array}{l}\text { Nombre de corps } \\
\text { rćcupérés dans l'utérus }\end{array}$} \\
\hline & $\begin{array}{c}\text { OEufs } \\
\text { de lapine }\end{array}$ & $\begin{array}{l}\text { Billes } \\
\text { de résine }\end{array}$ & & CEufs & Billes \\
\hline 870 & 7 & 0 & $2 \mathrm{~h}$ & 3 & 0 \\
\hline 656 & 0 & 13 & ' $\mathrm{h}: 30$ & 0 & 0 \\
\hline 931 & 8 & 0 & $\therefore 8 \mathrm{~h}$ & 0 & 0 \\
\hline 1031 & 7 & 0 & $3 \mathrm{mn}$ & 4 & 0 \\
\hline
\end{tabular}

Ces résultats médiocres s'accordent avec ceux de tous les auteurs qui ont tenté ce type de transplantation. La non récupération des corps transplantés s'expliquerait par l'existence de contractions utérines qui expulsent les œufs dans le vagin, sous l'influence des stimulations cervicales, vaginales ou vulvaires, provoquées par la manipulation. 
2. Inovulation par sonde à demeure.

Afin d'empêcher ce rejet des corps introduits, nous avons mis en place, le jour des chaleurs, une sonde munie d'un ballonnet terminal dont le gonflement permet l'ancrage de la sonde in utero. Ainsi, les œufs pouvaient être déposés au moment choisi sans stimulation des voies génitales.

Dans tous les essais, même en prenant toutes les précautions d'asepsie, une réaction infectieuse a pris place dans l'utérus.

\section{Inovulation avec dilatation utérine.}

Nous avons repris la technique de Rowson et Moor (Ig66) qui consiste à insuffler du gaz carbonique dans la corne en même temps que l'on dépose les " œufs ", le $\mathrm{CO}_{2}$ étant utilisé dans un but d'anesthésie locale et de relaxation.

Le tableau 2 résume nos résultats.

TABLEAU 2

Transfert via le cervix, avec dilatation utérine

\begin{tabular}{|c|c|c|c|c|c|c|c|c|}
\hline \multirow{2}{*}{$\begin{array}{c}\text { Délai } \\
\text { trans- } \\
\text { plantation- } \\
\text { abattage }\end{array}$} & \multicolumn{3}{|c|}{ Gufs de lapine } & \multicolumn{3}{|c|}{ Billes de résine } & \multicolumn{2}{|c|}{$\begin{array}{l}\text { Nombre d'animaux } \\
\text { avec récupération }>0\end{array}$} \\
\hline & $\begin{array}{l}\text { Nombre } \\
\text { animaux }\end{array}$ & $\begin{array}{l}\text { Nombre } \\
\text { oufs } \\
\text { transpl. }\end{array}$ & $\begin{array}{l}\text { Nombre } \\
\text { oufs } \\
\text { récupérés }\end{array}$ & $\begin{array}{l}\text { Nombre } \\
\text { animaux }\end{array}$ & $\begin{array}{c}\text { Nombre } \\
\text { billes } \\
\text { transpl. }\end{array}$ & $\begin{array}{c}\text { Nombre } \\
\text { billes } \\
\text { récupérées }\end{array}$ & avec œufs & avec billes \\
\hline 2 à 1 i h & t & 32 & 1 & ' & 50 & 26 & $1 / \mathrm{k}$ & $3 / 4$ \\
\hline 's à $6 \mathrm{~h} \ldots$ & 12 & $1 \div 0$ & 17 & 9 & $10 \prime_{t}$ & 70 & $3 / 12$ & $9 / 9$ \\
\hline 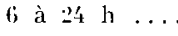 & 7 & 98 & 0 & 3 & 41 & 9 & $0 / 7$ & $3 / 3$ \\
\hline Totanx & 23 & 250 & 18 & 16 & 175 & 105 & $1 / 23$ & $15 / 16$ \\
\hline
\end{tabular}

Dans l'ensemble, les résultats sont meilleurs que ceux du premier essai, mais seules les billes sont récupérées dans des proportions acceptables, peut-être parce que plus facilement visibles ou parce que plus adhérentes à la muqueuse utérine, ce qui limiterait leur expulsion.

\section{B. - Inovulation par voie transutérine}

Nous avons très rapidement abandonné la technique de Sugre (I965) à cause des difficultés pratiques et parce qu'elle n'évite pas les stimulations vaginales, ni complètement les stimulations cervicales. Cette technique consiste en effet à perforer le vagin au niveau du cul de sac de Douglas à l'aide d'une longue aiguille puis à pénétrer dans l'utérus pour y déposer les œufs. Le cervix et les cornes utérines sont maintenues par palper transrectal.

Ceci nous a conduit à mettre au point une technique de transplantation à travers la paroi abdominale ce qui évite les stimulations génitales et l'infection que favorise la voie transvaginale ou transcervicale. 
Une anesthésie locale ( $50 \mathrm{ml}$ de tutocaïne à $2 \mathrm{p}$. roo) permet la pénétration, au niveau de la fosse paralombaire, d'un endoscope dans le canal duquel on introduit l'appareil à transplanter.

Ia cavité utérine étant virtuelle, la difficulté est d'assurer la position intraluminale de l'extrémité distale de l'aiguille qui guide le cathéter contenant les œufs. Cette position était assurée dans un premier appareil par un ballonnet gonflé in utero puis, dans un second, par la dilation utérine obtentie par simple injection de gaz carbonique. Le dispositif actuellement expérimenté est d'une manipulation simplifiée : après que 1'aiguille ait pénétré le myomètre, le cathéter en téflon contenant les œufs, projeté sous la pulsion d'un ressort, traverse la muqueuse utérine jusqu'à la lumière dans laquelle il s'oriente, du fait de sa flexibilité propre.

L'intervention totale, pratiquée dans des conditions aseptiques, demande une vingtaine de minutes.

I 4 vaches ont reçu in utero, 3 à 8 jours après les chaleurs, I à 4 œufs (stade 8 cellules-morula) récupérés par perfusion in vitro du tractus des vaches donneuses I à 3 heures avant l'intervention et conservés dans du liquide folliculaire autologue ou du milieu 'TC I99:

I 3 sont revenues en chaleurs I 8 à 25 jours après l'œstrus précédent et I, 40 jours après. On ignore les causes de cet échec qui pourrait être dû, soit à la qualité des cufs transplantés (dépréciation entre l'abattage de la donneuse et la récupération, ou au cours de la conservation), soit au transfert lui-même.

\section{II. - DISCUSSION}

Les auteurs s'accordent en général sur les causes qui expliquent les mauvais résultats obtenus à la suite des transplantations par la voie transvaginale.

\section{A. - L'expulsion de l'ceuf hors de l'utérus \\ à la suite des stimulations génitales}

HAYs et VANDEMARK (I953) ont montré que la Vache répond aux stimulations du tractus génital par une décharge dans l'organisme d'une substance à action ocytocique et Rowson, BeNNeTT et HARPER (I964), ne peuvent bloquer les contractions utérines responsables de l'expulsion dans le vagin de particules placées in utero, malgré 1'utilisation de diverses drogues (adrénaline, atropine, procaïne, papavérine, anesthésie épidurale).

OTsuki et Soma (I969) obtiennent des résultats comparables chez la chèvre. C'est seulement en insufflant du gaz carbonique dans l'utérus de génisses lors du transfert que HAFEZ et SUGIE (I963) retrouvent in utero I 3 p. roo des cufs de lapine transplantés is à $48 \mathrm{~h}$ plus tôt (aucune récupération s'ils n'insufflent pas l'utérus avec $\mathrm{CO}_{2}$ ).

L'emploi du $\mathrm{CO}_{2}$ (Rowson et MoOR, I956, puis Vincent, Milis et Rundeit, r 969) a permis l'amélioration des résultats par transfert transcervical ; mais le succès reste limité et nos propres essais (tab1. 2) montrent que dans des délais brefs ( 2 à 4 h), 
les œufs de Lapine sont rarement retrouvés dans l'utérus de la Vache, les résultats n'étant pas supérieurs à ceux obtenus sans insufflation (tabl. I).

Dans une expérience sur la Lapine (TESTART, I969), nous avons montré que les stimulations génitales n'entraînent pas l'expulsion des œufs transplantés, ce qui implique une relative spécificité du phénomène observé chez la Vache.

\section{B. - L'infection utérine}

Elle est provoquée par l'introduction in utero de matériaux septiques chez l'animal au post-œstrus. Dziuk et al. (I958), en injectant dans 1'utérus de vaches fécondées, du sérum physiologique récupéré après perfusion d'utéri d'autres femelles n'observent que 2 gestations sur I4 essais, mais 5 gestations sur 8 essais s'ils ajoutent de la pénicilline à ce sérum physiologique. On connait la plus faible résistance à l'infection utérine de la femelle en phase progestative par rapport à la phase œestrogénique dans pluseurs espèces comme la Vache (Rowson, LAMMING et FRY, I953), la Brebis (BRINSFIELD HAWK et CEFFEL, I967), la Lapine (BLACK et al, I953 ; BroOME et LAMMING, I958 ; BROOME, LAMming et SMITH, I959). Les stéroïdes ovariens joueraient un rôle déterminant dans cette résistance, en agissant soit sur le nombre et la vitesse de mobilisation des leucocytes, soit sur la capacité antibactérienne de ces leucocytes, soit sur le drainage de l'utérus.

En plaçant une sonde à demeure in utero, nous avons vérifié la susceptibilité de l'utérus aux germes infectieux. Les expériences menées chez la Lapine ('TESTART, r969) ont montré que l'influence défavorable du passage transcervical du cathéter de transplantation sur le résultat du transfert (tabl. 3) est dû en partie à l'infection du tractus génital après cette manipulation ; chez les seuls animaux à tractus normal, (47,5 p. roo) le faible taux d'implantation (45,I p. Ioo contre $77, \mathrm{I}$ p. Ioo pour la voie transpariétale) peut être la conséquence d'une contamination bactérienne très localisée atteignant seulement les blastocystes.

TABLEAU 3

Transplantation par voies transcervicale ou transpariétale de blastocystes dans l'utérus de la Lapine au $4^{\mathrm{e}}$ jour post-coïtum

\begin{tabular}{|c|c|c|c|c|c|c|}
\hline \multirow[b]{2}{*}{$\begin{array}{c}\text { Type de } \\
\text { transplan- } \\
\text { tation }\end{array}$} & \multirow[b]{2}{*}{$\begin{array}{l}\text { Nombre de } \\
\text { femelles } \\
\text { opérées }\end{array}$} & \multirow[b]{2}{*}{$\mid \begin{array}{c}P .100 \\
\text { gestantes à } \\
11 \text { jours }\end{array}$} & \multirow[b]{2}{*}{$\begin{array}{l}\text { P. } 100 \text { femelles } \\
\text { à tractus normal } \\
\text { (sans infection } \\
\text { ni adhérences) }\end{array}$} & \multicolumn{3}{|c|}{ Animaux à tractus normal } \\
\hline & & & & $\begin{array}{c}\text { Nombre } \\
\text { blastocystes } \\
\text { transplantés }\end{array}$ & $\begin{array}{c}\text { P. } 100 \\
\text { implantation } \\
\text { (intervalle } \\
\text { de variation) }\end{array}$ & $\begin{array}{l}\text { P. } 100 \text { survie } \\
\text { à } 11 \text { jours }\end{array}$ \\
\hline $\begin{array}{c}\text { Voie trans } \\
\text { cervicale }\end{array}$ & 40 & $60 \quad(2 / 4)$ & $47,5 \quad(19)$ & 233 & $45,1 \quad(0-100)$ & $88,6 \quad(93)(1)$ \\
\hline pariétale & 35 & $85,7 \quad(30)$ & $91,4 \quad(32)$ & 375 & $77,1 \quad(10-100)$ & $86,5(250)(1)$ \\
\hline
\end{tabular}

(1) Rapporté au nombre de blastocystes implantés. 


\title{
CONCLUSION
}

Les résultats expérimentaux rapportés montrent qu'une technique de transplantation des œufs chez les Bovins doit tenir compte de deux facteurs essentiels. La susceptibilité de l'utérus en état progestatif à la pollution par des matériaux septiques et la réactivité myométriale aux stimulations génitales.

La voie transpariétale de transfert des œufs élimine ces deux difficultés. Les résultats récents de Rowson, Moor et Lawson (I969) montrent que si l'œuf fécondé de vache est placé chirurgicalement dans l'utérus au $4^{\mathrm{e}}$ ou $5^{\mathrm{e}}$ jour du cycle, dans les meilleurs conditions (récupération in vivo des œufs, milieu de perfusion et de conservation convenable) on obtient plus de 90 p. roo de receveuses gestantes. Ils montrent également que l'œuf peut être conservé in vitro au moins 2 heures avant transfert et que les cycles de la donneuse et de la receveuse peuvent être synchronisés à 2 jours près.

Il reste à simplifier cette méthode. La technique transpariétale que nous expérimentons parce qu'elle ne nécessite qu'une anesthésie locale et une plage d'intervention chirurgicale réduite nous semble susceptible d'application pratique.

\section{RÉSUMÉ}

En comparant diverses techniques pratiques de transplantation des oufs fécondes in utero chez la Vache, nous avons retrouvé les difficultés rapportées par la bibliographje et qui tiennent à deux phénomènes: l'expulsion de l'œuf dans le vagin conséquemment aux stimulations géni. tales (que nous n'avons pas rencontrée chez la Lapine) et l'infection utérine.

Aussi, nous expérimentons une méthode transabdominale qui devtait permettre cl'obtenir un succès analogue à celui de Rowson, Mook et LAwson (Ig69) par intervention chirurgicale, tout en étant susceptible d'application pratique.

Nous n'avons pas obtenu de gestation jusqu'à ce jour.

\author{
SUMMARY
}

\section{SEARCH FOR A METHOD OF EGG TRANSFER IN THE COW}

Comparing various practical techniques of transplanting fertilized eggs in utero in the cow, we cncountered the same difficulties as those mentioned in the literature; namely, expulsion of the egg into the vagina following genital stimulation (which we did not obscrve in the rabbit), and uterine infection.

We are also studying a transabdominal method which would be practically applicable, and with which we hope to obtain success analogous to that of Rowson, Moor and LAwsos (Ig69) who employed surgical intervention.

Up to now, we have not obtained a pregnancy. 


\section{RÉFÉRENCES BIBLIOGRAPHIQUES}

Avery T. L., Fahning M. L., Pursel V. G., Graham E. F., I962. Investigations associated with the transplantation of bovine ova. IV. - Transplantation of ova. J. Reprod. Fert., 3, 229-238.

Black W. G., Simon J., McNutt S. H., Casida L. E., I953. Investigation on the physiological basis for the differential response of estrous and pseudopregnant rabbit uteri to induced infection. Amer. J. Vet. Res., 14, 318-323.

Brinsfield T. H., Hawk H. W., LefFel E. C., r967. Control by ovarian status of induced leukocytic responses in the sheep uterus. Amer. J. Vet. Res., 28, I27.

Broome A. W. J., Lamming G. E., 1958. The infuence of ovarian hormones on resistance to uterine infections. Vet. Rec., 70, 603-604.

Broome A. W. J., Lamming G. E., Smith W., I959. Studies on the relationship between ovarian hormones and uterine infection. IV. - The role of the granulocyte system in uterine defence. J. Endocr. 19, $274-281$.

Dzivk P. J., Donker J. I., Nichols J. R., Petersen W. E., 1958. Problems associated with the transfer of ova between cattle. Univ. Minnesota Agric. Exp. Sta. Tech. Bull., 222, 75 pp.

Hafez E. S. E., Sugie T., I963. Reciprocal transfer of cattle and rabbit embryos. J. Anim. Sci., 22, $3 \mathrm{I}-35$.

HaYs R. L., VANDEMARK N. L., I953. Effect of stimulation of the reproductive organs of the cow on the release of an oxytocin-like substance. Endocrinology, 82, 157-r65.

Mutter L. R., Graden A. P., Olds D., ig65. Successful non surgical bovine embryo transfer. $A$. I. Digest, 12, (I I), 3 .

Otsukr K., Soma T., I964. Transfer of fertilized ova through the cervix in goats. Bull. Nat. Inst. Anim. Ind., 6, 27-32.

Rowson L. E. A., BennetT J. P., Harper M. J. R., I964. The problem of non-surgical egg transfer to the cow uterus. Vet. Rec., 76, 2I-23.

Rowson 1. E. A., LAmming G. E., FRY R. M., 1953. The relationship between ovarian hormones and uterine infection. Vet. Rec., 65, 335-343.

Rowson L. E. A., Moor R. M., 1966. Non-surgical transfer of cow eggs. J. Reprod. Fert., 1131 I3 I2.

Rowson L. E. A., Moor R. M., LAwson R. A. S., r969. Fertility following egg transfer in the cow; effect of method, medium and synchronization of westrus. J. Reprod. Fert., 18, 517-523.

Sugie T., 1965. Successful transfer of a fertilized bovine egg by non-surgical technique. J. Reprod. Fert., 10, I97-202.

Testart J., 1969. Comparaison de différentes techniques de transplantation des blastocystes chez la lapine. Ann. Biol. Anim. Bioch. Biophys., 9, 351-36o.

Umbaugh R. E., 1949. Superovulation and ovum transfer in cattle. Amer. J. Vet. Res., 37, $295-305$.

Vincent C. K., Mills A. C., Rundell J. W., I969. Non-surgical transfert of embryos in beef cattle. J. anim. Sci., 28, I47.

Willett E. L., Black W. G., Casida L. E., Stone W. H., Buckner P. J., I951. Successful transplantation of a fertilized bovine ovum. Science, 113, 247.

Willett E. L., Buckner P. J., Larson G. I., 1953. Three successful transplantations of fertilized bovine eggs. J. Dairy Sci., 39, 520-523. 